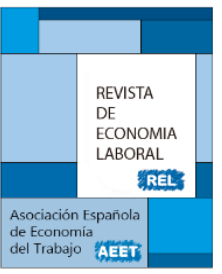

\title{
ANÁLISIS DE LAS INAPLICACIONES DE CONVENIOS TRAS LA REFORMA LABORAL ${ }^{1}$
}

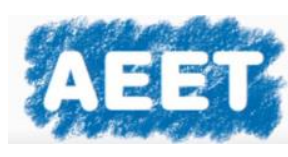

\author{
César Rodríguez Gutiérrez ${ }^{2}$ y Juan Francisco Canal Domínguez \\ Universidad de Oviedo
}

Recibido Septiembre 2016; Aceptado Noviembre 2016

\section{Resumen}

La reforma laboral de 2012 incorporó un mecanismo sencillo para la inaplicación de un convenio colectivo, con el objetivo de que las empresas en crisis pudieran ajustar los costes salariales para evitar recortes más profundos en el empleo. Desde esa fecha, las inaplicaciones no han sido muy frecuentes en la práctica, situándose el porcentaje de trabajadores afectados por las mismas por debajo del 1\% (con la excepción del año 2013, en que se elevó al 1,5\%). El análisis de una muestra de 447 empresas que inaplicaron un convenio colectivo en el periodo 2012-2013, ha permitido observar cómo, tras el profundo deterioro de los ingresos de explotación, los resultados y la rentabilidad económica que tuvo lugar al inicio de la crisis, los tres indicadores mencionados se han ido recuperando ligeramente a partir de 2012, primer año en que las inaplicaciones fueron posibles. Lo mismo ha sucedido (con un año de retraso) con el nivel de empleo, aunque no con el coste laboral medio, que sólo ha comenzado a recuperarse en 2014. También se ha podido constatar que las empresas que inaplican presentan una elasticidad del empleo ante cambios en los salarios de -0,37.

Palabras clave: Reforma laboral, Inaplicación de convenios colectivos

Clasificación JEL: J52, J53

${ }^{1}$ Este artículo se ha beneficiado de la financiación de los proyectos: ECO2013-43925-R, del Ministerio de Economía y Competitividad, y SV-15-GRUPUO-RULE, del Principado de Asturias.

${ }^{2}$ Autor para correspondencia: crodri@uniovi.es

C Revista de Economía Laboral 


\begin{abstract}
The 2012 labour reform includes a simple procedure for the non-application of collective agreements, so that companies in crisis could adjust labour costs to avoid deeper employment cuts. Non-applications have not been very frequent since then, being the percentage of workers affected by such new regulation below 1\% (except for 2013, when this percentage rose up to $1.5 \%$ ). The analysis of a sample of 447 firms which did not apply a collective agreement in the period 2012-2013, allows us to observe that, after the deep fall in sales revenue, profits and the index of economic profitability at the beginning of the economic crisis, these three indicators have been slightly recovering since 2012 (the first year when non-application of collective agreements was possible). The same has happened with the level of employment (with a lag of one year), but not with the average labour cost, that only increased in 2014. It has also been observed that firms which did not apply collective agreements present employment elasticity with respect to wages of -0.37.
\end{abstract}

Key words: Labour reform, Non-application of collective agreements

JEL Classification: J52, J53 


\section{Introducción}

La reforma laboral del año 2012 alteró de manera sustancial el modelo español de negociación colectiva. Hasta la aprobación de Real Decreto-ley 3/2012 de 10 de febrero y la Ley 3/2012 de 6 de julio, el sistema de negociación colectiva apenas había sufrido cambios en lo sustancial. Los rasgos más característicos del modelo eran la "eficacia general automática" de los convenios colectivos y el principio de aplicación del "convenio más favorable". Este marco daba lugar a un modelo de negociación claramente jerarquizado en tres niveles principales: los convenios de sector nacional, los convenios de sector provincial (y autonómico), y los convenios de empresa. Según se descendía de nivel, los convenios sólo podían mejorar las condiciones pactadas en el nivel superior, y el descuelgue de los convenios, aunque era una opción legal, no se llevaba a cabo en la práctica dadas las muy exigentes condiciones legales para su aplicación. ${ }^{3}$ Sin embargo, la reforma laboral estableció algunas modificaciones sustanciales en este modelo. Por un lado, la denominada "prioridad aplicativa" del convenio de empresa hace posible, por primera vez, que una empresa concreta pueda negociar un convenio colectivo propio fijando peores condiciones laborales y salariales que las establecidas en el convenio de sector que le sería de aplicación. ${ }^{4}$ En segundo lugar, la limitación temporal de la "ultraactividad" de los convenios acaba con la prórroga indefinida de los

\footnotetext{
3 En efecto, la posibilidad de descuelgue ya existía desde 1994, pero, como señala Thibault (2014), "tal posibilidad ha sido más teórica que real. En un primer momento, tanto las fuertes restricciones impuestas por los negociadores de las denominas «cláusulas de descuelgue», más pendientes se diría de mantener la aplicación del convenio a cualquier precio que de facilitar una salida a las empresas en crisis, como las propias dificultades para alcanzar un acuerdo en la empresa hicieron de la inaplicación del convenio algo inusual por casi irrealizable” (pág. 218). Segura (2001, pág. 177) y Bentolila y Jimeno (2002, pág. 8) llegan también a una conclusión similar. ${ }^{4}$ El artículo 14.3 de la Ley 3/2012 de 6 de julio, modifica el apartado 2 del artículo 84 del Texto Refundido de la Ley del Estatuto de los Trabajadores, aprobado por Real Decreto Legislativo 1/1995, de 24 de marzo, estableciendo que: "La regulación de las condiciones establecidas en un convenio de empresa, que podrá negociarse en cualquier momento de la vigencia de convenios colectivos de ámbito superior, tendrá prioridad aplicativa respecto del convenio sectorial estatal, autonómico o de ámbito inferior" en materias como la cuantía del salario base y de los complementos salariales, el abono o la compensación de las horas extraordinarias, y el horario y la distribución del tiempo de trabajo, entre otros. Como señala Del Rey Guanter (2014) "la Ley 3/2012 ha independizado -parcial, pero sustancialmente- al nivel de empresa de los niveles superiores, dotándole de una autonomía absoluta en determinadas materias respecto a lo que pueda disponerse en esos niveles" (pág. 205).
} 
mismos mientras se negocian los nuevos acuerdos. ${ }^{5}$ En tercer lugar, la ley establece por primera vez mecanismos sencillos para que una empresa pueda inaplicar el convenio colectivo que está obligada a cumplir. Los tres aspectos citados han podido contribuir desde 2012 a debilitar el poder de negociación de los trabajadores. Hemos de tener en cuenta que, con la normativa actual, ante una negociación complicada los empresarios poseen un mayor incentivo para alargar los plazos de la misma, e incluso, tras alcanzar un acuerdo, podrían descolgarse del mismo si se dan las circunstancias apropiadas. Este "efecto amenaza" (que es la posibilidad real de no disponer finalmente de un convenio colectivo) puede llevar a los trabajadores a firmar acuerdos menos satisfactorios que los alcanzables con la legislación anterior, y, sin duda, contribuye a facilitar y acelerar el proceso de ajuste salarial (devaluación interna) vivido por la economía española durante la crisis. ${ }^{6}$

El objetivo de este trabajo consiste en evaluar la intensidad del fenómeno de las inaplicaciones llevadas a cabo por las empresas españolas a partir de la reforma laboral, así como la utilidad de esta medida para recuperar a las empresas en crisis. Es decir, trataremos de saber, primero, cuántas empresas y de qué tipo, han inaplicado un convenio colectivo y, segundo, en qué medida la inaplicación les ha permitido recuperar ingresos, empleo y beneficios. Para ello, en la Sección 2 describiremos el marco legal de las inaplicaciones. A continuación, en la Sección 3 se estudiarán la frecuencia y los tipos de inaplicaciones llevadas a cabo entre 2012 y 2016. En la Sección 4 se analizará la evolución de los ratios económico-financieros de una muestra de empresas que han inaplicado un convenio colectivo en los años 2012 y 2013. Finalmente, en la Sección 5 se resumirán las principales conclusiones de la investigación.

\section{El marco legal de las inaplicaciones de convenios colectivos}

¿Cuáles son las circunstancias que la ley prevé para que una empresa pueda descolgarse de un convenio colectivo? El artículo 14 de

\footnotetext{
5 Es preciso señalar que la cuestión de la ultraactividad está sujeta a bastante discusión. Así, por sentencia de 22 de diciembre de 2014, el Pleno de la Sala de lo Social del Tribunal Supremo establece que las condiciones pactadas en un convenio extinguido podrían incorporarse al contrato individual de trabajo, lo que limita los efectos jurídicos y económicos del fin de la ultraactividad.

${ }^{6}$ Véase, en este mismo sentido, Pérez Infante (2015, págs. 16-17).
} 
la Ley 3/2012, de 6 de julio, de medidas urgentes para la reforma del mercado laboral, modifica el apartado 3 del artículo 82 del Texto Refundido de la Ley del Estatuto de los Trabajadores, estableciendo que:

"Cuando concurran causas económicas, técnicas, organizativas o de producción, por acuerdo entre la empresa y los representantes de los trabajadores legitimados para negociar un convenio colectivo conforme a lo previsto en el artículo 87.1, se podrá proceder, previo desarrollo de un periodo de consultas en los términos del artículo 41.4, a inaplicar en la empresa las condiciones de trabajo previstas en el convenio colectivo aplicable, sea este de sector o de empresa, que afecten a las siguientes materias:

a) Jornada de trabajo.

b) Horario y la distribución del tiempo de trabajo.

c) Régimen de trabajo a turnos.

d) Sistema de remuneración y cuantía salarial.

e) Sistema de trabajo y rendimiento.

f) Funciones, cuando excedan de los límites que para la movilidad funcional prevé el artículo 39 de esta Ley.

g) Mejoras voluntarias de la acción protectora de la Seguridad Social”.

Además, “...se entiende que concurren causas económicas cuando de los resultados de la empresa se desprenda una situación económica negativa, en casos tales como la existencia de pérdidas actuales o previstas, o la disminución persistente de su nivel de ingresos ordinarios o ventas. En todo caso, se entenderá que la disminución es persistente si durante dos trimestres consecutivos el nivel de ingresos ordinarios o ventas de cada trimestre es inferior al registrado en el mismo trimestre del año anterior".

Obsérvese que la ley es muy clara en cuanto al establecimiento de las condiciones para el descuelgue. Por un lado, basta con analizar la evolución de las ventas o ingresos de explotación de la empresa, información objetiva que figura en los libros de contabilidad. Adicionalmente, es suficiente con que se reduzcan las ventas durante dos trimestres consecutivos con respecto a los mismos trimestres del año anterior. Si esta caída se produce (algo habitual en un periodo de crisis como el vivido recientemente por la economía española), entonces la empresa se podría descolgar de las tablas salariales del convenio y de un conjunto de elementos importantes como la jornada, el horario, los sistemas de trabajo, etc. Desde el punto de vista salarial, teóricamente el único límite inferior para estas empresas que se descuelgan lo 
constituye el Salario Mínimo Interprofesional (SMI) fijado anualmente por el Gobierno.

\section{Evolución de las inaplicaciones: cuantía y tipos}

En este apartado se ofrecen algunas cifras referidas a las inaplicaciones de convenios colectivos desde el año 2012. Para este año, la Estadística de Convenios Colectivos del Ministerio de Empleo y Seguridad Social proporciona sólo la cifra total de inaplicaciones realizadas entre los meses de marzo y diciembre, que suma un total de 748, afectando a 29.352 trabajadores. A partir del año 2013 la cifra total de inaplicaciones se desglosa según diversas variables (como el tamaño de la empresa, el sector...). Dicha información aparece resumida en los cuadros 2 a 4 . Es preciso señalar que la información correspondiente a 2013 y 2014 se ha extraído de las estadísticas definitivas de cada año, mientras que las cifras de 2015 no son definitivas, sino un avance, y las de 2016 son incompletas (pues se refieren al periodo comprendido entre enero y abril de dicho año).

Como paso previo, y con el fin de ofrecer una primera fotografía de la estructura de la negociación colectiva y de su evolución reciente, en el cuadro 1 se presentan las cifras correspondientes al número de convenios firmados, empresas y trabajadores afectados, para el periodo 2012-2016.

El hecho de que los datos de 2015 y 2016 no sean definitivos impide comparar correctamente esas cifras con las anteriores. No obstante, la observación de los datos correspondientes a los años 2012, 2013 y 2014, parece indicar que se ha producido un incremento significativo en el número de convenios firmados tras la aprobación de la reforma laboral de 2012. En ese sentido, es posible que la reforma haya logrado cumplir uno de los objetivos que perseguía: descentralizar nuestro modelo de negociación colectiva. En concreto, el número de convenios firmados en el ámbito de la empresa creció un $23,6 \%$ en ese periodo, si bien el aumento en el número de trabajadores afectados por ese tipo de convenios fue muy inferior (tan sólo del 2,9\%). Eso hace que realmente no se pueda hablar con rotundidad de un cambio en la estructura de la negociación colectiva, sobre todo, teniendo en cuenta que el porcentaje de trabajadores cubiertos por convenios de ámbito superior a la empresa también creció en una proporción ligeramente inferior (1,9\%). En relación a la distribución sectorial de los convenios, los datos resaltan la prevalencia de las negociaciones en el sector servicios (tanto en términos de número de convenios, como de empresas 
y trabajadores afectados), seguido por la industria, la construcción y el sector agrario.

Cuadro 1: Convenios, empresas y trabajadores afectados, por ámbito funcional y sector de producción, y año de firma.

\begin{tabular}{|c|c|c|c|c|c|}
\hline & \multicolumn{5}{|c|}{ Ámbito funcional } \\
\hline & 2012 & 2013 & 2014 & $2015(p)$ & 2016(a) \\
\hline \multicolumn{6}{|l|}{ Total } \\
\hline Convenios & 4.376 & 4.589 & 5.185 & 4.460 & 1.385 \\
\hline Empresas & 1.161 .958 & 1.312 .906 & 1.436.946 & 1.069.772 & 468.376 \\
\hline Trabajadores & 10.099 .019 & 10.265 .402 & 10.304 .700 & 7.818 .244 & 4.405 .980 \\
\hline \multicolumn{6}{|c|}{ Convenios de empresa y grupo de empresas } \\
\hline Convenios & 3.335 & 3.506 & 4.122 & 3.768 & 1.052 \\
\hline Trabajadores & 1.112 .734 & 1.100 .292 & 1.144 .770 & 988.483 & 352.249 \\
\hline \multicolumn{6}{|c|}{ Convenios de otro ámbito } \\
\hline Convenios & 1.041 & 1.083 & 1.063 & 692 & 333 \\
\hline \multirow[t]{3}{*}{ Trabajadores } & 8.986 .285 & 9.165 .110 & 9.159 .930 & 6.829 .761 & 4.053 .731 \\
\hline & \multicolumn{5}{|c|}{ Sector de producción } \\
\hline & 2012 & 2013 & 2014 & 2015(a) & 2016(p) \\
\hline \multicolumn{6}{|l|}{ Agrario } \\
\hline Convenios & 59 & 62 & 78 & 70 & 33 \\
\hline Empresas & 82.997 & 78.945 & 127.053 & 106.002 & 43.876 \\
\hline Trabajadores & 779.746 & 644.084 & 743.809 & 570.152 & 339.526 \\
\hline \multicolumn{6}{|l|}{ Industria } \\
\hline Convenios & 1.743 & 1.801 & 1.942 & 1.620 & 525 \\
\hline Empresas & 206.838 & 193.261 & 177.254 & 158.960 & 101.912 \\
\hline Trabajadores & 2.255 .437 & 2.334 .772 & 2.343 .905 & 2.191 .094 & 1.369 .864 \\
\hline \multicolumn{6}{|l|}{ Construcción } \\
\hline Convenios & 103 & 109 & 127 & 135 & 32 \\
\hline Empresas & 111.043 & 129.074 & 104.537 & 96.249 & 371 \\
\hline Trabajadores & 741.055 & 867.697 & 815.595 & 695.853 & 5.256 \\
\hline \multicolumn{6}{|l|}{ Servicios } \\
\hline Convenios & 2.471 & 2.617 & 3.038 & 2.635 & 795 \\
\hline Empresas & 761.080 & 911.626 & 1.028 .102 & 708.561 & 322.217 \\
\hline Trabajadores & 6.322 .781 & 6.418 .849 & 6.401 .391 & 4.361 .145 & 2.691 .334 \\
\hline
\end{tabular}

(a)Avance (p) Provisional

Fuente: Estadística de Convenios Colectivos. Ministerio de Empleo y Seguridad

Social. Elaboración propia

Por su parte, el cuadro 2 muestra la información sobre el número de inaplicaciones, las empresas y los trabajadores afectados por las mismas para el periodo 2013-2016, en términos absolutos y también como porcentaje del total de empresas y trabajadores sujetos a convenio. A la vista de estos datos, se puede concluir que la utilización de este instrumento de flexibilidad por parte de las empresas ha sido 
bastante residual, ya que las cifras se sitúan por debajo del $1 \%$, a excepción del porcentaje de trabajadores afectados en el año 2013, que llegó al 1,5\%. Además, y a la espera de la consolidación de las cifras provisionales de los años 2015 y 2016, parece observarse una tendencia decreciente en el uso de este recurso por parte de las empresas, si atendemos al porcentaje de trabajadores afectados. Este resultado es bastante lógico, por cuanto la recuperación iniciada a partir de 2014 ha dado lugar a un aumento generalizado en las ventas de las empresas que impide cumplir las condiciones necesarias establecidas en la ley para llevar a cabo el descuelgue de un convenio colectivo.

Cuadro 2: Inaplicaciones de convenios, empresas y trabajadores. Entre paréntesis, porcentaje en relación al total de empresas y trabajadores cubiertos por la negociación colectiva.

\begin{tabular}{lccc}
\hline & Inaplicaciones & Empresas afectadas & Trabajadores \\
\hline $\mathbf{2 0 1 3}$ & 2.512 & 2.179 & 159.550 \\
& & $(0,17 \%)$ & $(1,55 \%)$ \\
$\mathbf{2 0 1 4}$ & 2.073 & 1.831 & 66.203 \\
& & $(0,13 \%)$ & $(0,64 \%)$ \\
$\mathbf{2 0 1 5 ( a )}$ & 1.437 & 1.254 & 43.173 \\
& & $(0,12 \%)$ & $(0,55 \%)$ \\
$\mathbf{2 0 1 6 ( p )}$ & 600 & 556 & 11.259 \\
& & $(0,12 \%)$ & $(0,26 \%)$ \\
\hline
\end{tabular}

(a)Avance (p) Provisional

Fuente: Estadística de Convenios Colectivos. Ministerio de Empleo y Seguridad Social. Elaboración propia

En el cuadro 3 se desglosa el número de inaplicaciones por sector de actividad y tamaño de la empresa. En relación al sector, lógicamente los servicios y la industria resultan mayoritarios por ese orden, si bien, a lo largo del tiempo ha habido un cambio de composición en cuanto al porcentaje de trabajadores afectados, creciendo notablemente la cifra de trabajadores pertenecientes a la industria y la construcción, y cayendo la correspondiente a los servicios. En concreto, los servicios pasan de aglutinar al 81,2\% de los afectados en 2013, al 65,3\% en 2016 (casi 16 puntos porcentuales menos); mientras que el porcentaje de trabajadores que pertenecen al sector industrial crece en casi 8 puntos (del $13,4 \%$ al $21,3 \%$ ), y el correspondiente a la construcción aumenta del 0,8\% al 7,5\%. 
Según el tamaño de las empresas, el cuadro 3 muestra con claridad que son las empresas pequeñas (de menos de 50 trabajadores) las que han recurrido en mayor medida a este recurso y, además, su importancia es creciente en el tiempo (pasando del $81 \%$ al $88 \%$ del total entre 2013 y 2016). Sin embargo, la cifra más alta de trabajadores afectados por inaplicaciones corresponde a las empresas de mayor tamaño (de 250 trabajadores o más), excepto en el año 2016 (si bien, hay que tener en cuenta que las cifras correspondientes a este año son provisionales). En todo caso, parece observarse una tendencia a la disminución de la proporción de trabajadores afectados por inaplicaciones en las empresas grandes y al aumento en las pequeñas.

Por último, en el cuadro 4 se detalla la distribución de las inaplicaciones y de los trabajadores afectados en función de las condiciones de trabajo inaplicadas, del procedimiento de inaplicación y del ámbito funcional. En relación a las primeras, se puede observar que la inaplicación de los acuerdos en materia salarial (cuantía, sistema de remuneración o ambas asociadas a otros factores) constituye el motivo más frecuente, pues supone alrededor del 90\% de los casos. En cuanto al procedimiento elegido para la inaplicación, en la gran mayoría de los casos (más del 90\%) se llevó a cabo la inaplicación mediante un acuerdo en periodo de consultas. Por último, en relación al ámbito del convenio afectado, en la práctica totalidad de los casos las empresas han inaplicado aspectos relacionados con convenios de ámbito superior a la misma. En cuanto al porcentaje de trabajadores afectados, si bien la inaplicación de condiciones del propio convenio de la empresa afectaba a un significativo $25 \%$ de los trabajadores sujetos a inaplicaciones en el año 2013, este porcentaje ha ido reduciéndose de forma constante hasta suponer tan sólo el 8,8\% en el año 2016.

En resumen, las cifras ofrecidas por las estadísticas de convenios colectivos de trabajo indican que el recurso de las empresas a la inaplicación de condiciones pactadas en convenio propio o de ámbito superior, ha sido hasta el momento una práctica muy minoritaria (casi residual) por parte de las empresas, si atendemos al porcentaje de empresas y trabajadores afectados. Un análisis detallado de la casuística revela que las inaplicaciones afectan especialmente a empresas y trabajadores del sector servicios, y que si bien son las empresas de tamaño más pequeño las que más recurren a la inaplicación, el mayor porcentaje de trabajadores afectados tiene su puesto de trabajo en empresas de tamaño grande, aunque las diferencias con el grupo de trabajadores de empresas pequeñas se van recortando de forma ostensible. En términos cualitativos, la condición más inaplicada por las empresas es la cuantía salarial, y el 
procedimiento elegido más extendido es el acuerdo en periodo de consultas. Por último, la mayor parte de las inaplicaciones se refieren a contenidos de convenios colectivos de ámbito superior a la empresa. 
Cuadro 3: Inaplicaciones de convenios (valor absoluto), empresas y trabajadores (porcentaje sobre el total de empresas y trabajadores afectados por las inaplicaciones).

\begin{tabular}{|c|c|c|c|c|c|c|c|c|c|}
\hline & & \multicolumn{4}{|c|}{ Sector } & \multicolumn{4}{|c|}{ Tamaño de la empresa } \\
\hline & & Agrario & Industria & Construcción & Servicios & $\begin{array}{c}1-49 \\
\text { trabajadores }\end{array}$ & $\begin{array}{c}50-249 \\
\text { trabajadores }\end{array}$ & $\begin{array}{l}250 \text { o más } \\
\text { trabajadores }\end{array}$ & $\begin{array}{c}\text { No } \\
\text { consta }\end{array}$ \\
\hline \multirow{4}{*}{$\begin{array}{l}\text { Inaplicaciones } \\
\text { de convenios }\end{array}$} & 2013 & 54 & 393 & 108 & 1.957 & 1.965 & 313 & 189 & 45 \\
\hline & 2014 & 64 & 298 & 139 & 1.572 & 1.690 & 274 & 68 & 41 \\
\hline & $2015(p)$ & 40 & 205 & 116 & 1.076 & 1.176 & 184 & 50 & 27 \\
\hline & 2016(a) & 13 & 78 & 48 & 461 & 517 & 64 & 9 & 10 \\
\hline \multirow[t]{4}{*}{ Empresas } & 2013 & $2,02 \%$ & $15,56 \%$ & $4,45 \%$ & $77,97 \%$ & $81,23 \%$ & $11,89 \%$ & $4,96 \%$ & $1,93 \%$ \\
\hline & 2014 & $2,89 \%$ & $15,02 \%$ & $6,94 \%$ & $75,15 \%$ & $83,40 \%$ & $11,85 \%$ & $2,57 \%$ & $2,18 \%$ \\
\hline & 2015(a) & $2,63 \%$ & $14,67 \%$ & $8,45 \%$ & $74,24 \%$ & $84,45 \%$ & $11,32 \%$ & $2,15 \%$ & $2,07 \%$ \\
\hline & 2016(p) & $2,34 \%$ & $13,49 \%$ & $8,27 \%$ & $75,90 \%$ & $87,95 \%$ & $8,99 \%$ & $1,26 \%$ & $1,80 \%$ \\
\hline \multirow[t]{4}{*}{ Trabajadores } & 2013 & $4,60 \%$ & $13,40 \%$ & $0,83 \%$ & $81,17 \%$ & $13,37 \%$ & $16,11 \%$ & $68,51 \%$ & $2,01 \%$ \\
\hline & 2014 & $9,61 \%$ & $13,09 \%$ & $2,66 \%$ & $74,65 \%$ & $26,79 \%$ & $30,74 \%$ & $41,26 \%$ & $1,21 \%$ \\
\hline & 2015(a) & $10,18 \%$ & $22,78 \%$ & $3,43 \%$ & $63,62 \%$ & $26,43 \%$ & $30,35 \%$ & $42,03 \%$ & $1,19 \%$ \\
\hline & 2016(p) & $5,93 \%$ & $21,25 \%$ & $7,51 \%$ & $65,32 \%$ & $41,76 \%$ & $36,02 \%$ & $18,51 \%$ & $3,71 \%$ \\
\hline
\end{tabular}

(a)Avance (p) Provisional

Fuente: Estadística de Convenios Colectivos. Ministerio de Empleo y Seguridad Social. Elaboración propia 
Cuadro 4: Inaplicaciones de convenios y trabajadores (porcentaje de trabajadores sobre el total de trabajadores afectados por las inaplicaciones), por condiciones de trabajo inaplicadas, procedimiento de inaplicación y ámbito funcional del convenio inaplicado.

\begin{tabular}{|c|c|c|c|c|c|c|c|c|}
\hline & \multicolumn{4}{|c|}{ Inaplicaciones } & \multicolumn{4}{|c|}{ Trabajadores } \\
\hline & 2013 & 2014 & 2015(a) & $2016(p)$ & 2013 & 2014 & 2015(a) & $2016(p)$ \\
\hline \multicolumn{9}{|l|}{ Condiciones de trabajo inaplicadas } \\
\hline Cuantía salarial & $66,56 \%$ & $64,30 \%$ & $62,21 \%$ & $63,33 \%$ & $42,01 \%$ & $60,16 \%$ & $52,66 \%$ & $43,95 \%$ \\
\hline Cuantía salarial y sistema de remuneración & $10,35 \%$ & $14,76 \%$ & $13,64 \%$ & $10,67 \%$ & $8,56 \%$ & $7,49 \%$ & $7,79 \%$ & $8,70 \%$ \\
\hline Cuantía salarial y jornada de trabajo & $3,46 \%$ & $3,33 \%$ & $3,13 \%$ & $5,33 \%$ & $4,67 \%$ & $4,20 \%$ & $4,20 \%$ & $4,47 \%$ \\
\hline Sistema de remuneración & $3,07 \%$ & $5,07 \%$ & $4,66 \%$ & $5,67 \%$ & $1,16 \%$ & $3,74 \%$ & $3,00 \%$ & $3,58 \%$ \\
\hline $\begin{array}{l}\text { Cuantía salarial, jornada de trabajo y } \\
\text { horario y distribución del tiempo de trabajo* }\end{array}$ & $2,23 \%$ & $1,74 \%$ & $1,39 \%$ & $1,50 \%$ & $5,04 \%$ & $10,58 \%$ & $1,03 \%$ & $2,28 \%$ \\
\hline $\begin{array}{l}\text { Cuantía salarial, sistema de remuneración, } \\
\text { jornada de trabajo y horario y distribución } \\
\text { del tiempo de trabajo** }\end{array}$ & $2,23 \%$ & $1,69 \%$ & $1,32 \%$ & $1,50 \%$ & $1,06 \%$ & $1,48 \%$ & $1,13 \%$ & $3,30 \%$ \\
\hline $\begin{array}{l}\text { Cuantía salarial y mejoras voluntarias de la } \\
\text { acción protectora de la Seguridad Social*** }\end{array}$ & $1,71 \%$ & $1,01 \%$ & $2,50 \%$ & $3,50 \%$ & $1,67 \%$ & $0,70 \%$ & $0,89 \%$ & $1,51 \%$ \\
\hline Resto de casos & $10,39 \%$ & $8,10 \%$ & $11,13 \%$ & $8,50 \%$ & $35,82 \%$ & $11,65 \%$ & $29,29 \%$ & $32,21 \%$ \\
\hline \multicolumn{9}{|l|}{ Procedimiento de inaplicación } \\
\hline Acuerdo en periodo de consultas & $90,72 \%$ & $95,80 \%$ & $90,61 \%$ & $91,67 \%$ & $78,83 \%$ & $91,48 \%$ & $88,46 \%$ & $89,37 \%$ \\
\hline $\begin{array}{l}\text { Acuerdo de la comisión paritaria del } \\
\text { convenio }\end{array}$ & $4,94 \%$ & $2,03 \%$ & $4,94 \%$ & $5,33 \%$ & $4,32 \%$ & $3,37 \%$ & $5,16 \%$ & $6,00 \%$ \\
\hline Acuerdo de mediación en órgano bipartito & $3,03 \%$ & $1,40 \%$ & $2,64 \%$ & $2,50 \%$ & $8,59 \%$ & $3,08 \%$ & $3,82 \%$ & $3,38 \%$ \\
\hline Laudo en órgano bipartito & $0,52 \%$ & $0,19 \%$ & $0,35 \%$ & $0,00 \%$ & $0,27 \%$ & $0,45 \%$ & $0,30 \%$ & $0,00 \%$ \\
\hline Decisión en el seno de un órgano tripartito & $0,80 \%$ & $0,58 \%$ & $1,46 \%$ & $0,50 \%$ & $7,99 \%$ & $1,61 \%$ & $2,27 \%$ & $1,24 \%$ \\
\hline \multicolumn{9}{|l|}{$\begin{array}{l}\text { Ámbito funcional del convenio } \\
\text { inaplicado }\end{array}$} \\
\hline Convenio de empresa & $5,10 \%$ & $3,09 \%$ & $2,92 \%$ & $2,33 \%$ & $25,09 \%$ & $13,31 \%$ & $15,53 \%$ & $8,83 \%$ \\
\hline
\end{tabular}




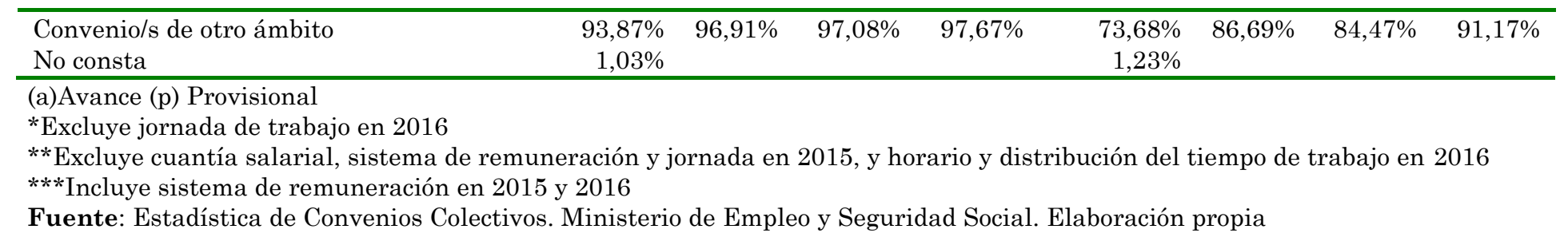




\section{Evolución de los indicadores económicos de las empresas que inaplican}

\subsection{Análisis descriptivo de la muestra de empresas}

En esta sección se analizará la evolución de algunos indicadores económicos de las empresas que han inaplicado un convenio colectivo a lo largo de los años 2012 y 2013. Con este objetivo, a partir de la información del Registro de Convenios Colectivos del Ministerio de Empleo y Seguridad Social, se ha seleccionado una muestra de empresas para las que se han obtenido los datos de ingresos de explotación, resultados después de impuestos, gastos de personal, número de empleos, salario medio, así como el índice de rentabilidad económica (definido como el cociente entre los resultados ordinarios antes de impuestos y el activo total), para el periodo 2007-2014. ${ }^{7}$ Esta información se ha obtenido de la base SABI (Sistema de Análisis de Balances Ibéricos). El periodo de estudio elegido coincide con el de crisis económica, siendo los objetivos de este análisis comprobar, en primer lugar, en qué medida los distintos indicadores económicos de las empresas que han inaplicado un convenio se han ido deteriorando desde el inicio de la crisis hasta llegar a hacer inevitable la inaplicación del mismo; y, en segundo lugar, si dicha inaplicación ha contribuido o no a sanear posteriormente esa situación, impulsando los ingresos y el empleo.

La muestra elegida está compuesta por 447 empresas, de las cuales $105(23,5 \%)$ recurrieron a la inaplicación en el año 2012 y 342 $(76,5 \%)$ lo hicieron en el año $2013 .{ }^{8}$ Como se puede apreciar en los gráficos 1 y 2 , la mayoría de las empresas pertenecen al sector servicios $(63,1 \%)$, seguido a gran distancia por la industria (23,5\%) y la construcción $(10,5 \%)$. En cuanto al tamaño, predomina la pequeña empresa (casi el $75 \%$ tiene menos de 50 trabajadores en plantilla), y,

\footnotetext{
${ }^{7}$ Es importante señalar que la muestra sólo contiene información de las empresas que han inaplicado el convenio en el periodo considerado. Como estas empresas suponen menos del 1 por ciento del empleo total de la economía española (excepto en 2013), podría considerarse que los indicadores del conjunto de la economía española constituyen el grupo de control con el que comparar, por ejemplo, la evolución de los ingresos de explotación o de los costes laborales, como se verá más adelante.

8 Hemos de señalar también que las 447 empresas para las que se dispone de información, son empresas "supervivientes" de la crisis, lo que sin duda, puede condicionar de algún modo los resultados.
}

(C) Revista de Economía Laboral 
sobre todo, la microempresa (menos de 10 trabajadores), que aglutina a cerca de la mitad de las empresas de la muestra. En cuanto a la distribución territorial, no existe un patrón definido, si bien es destacable que el $16 \%$ de las empresas están radicadas en la Comunidad Valenciana.

\section{Gráfico 1: Distribución de las empresas de la muestra según sector de actividad.}

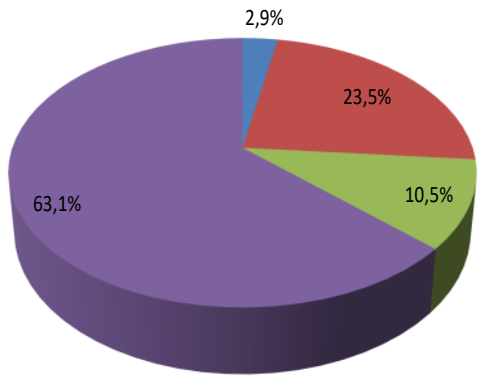

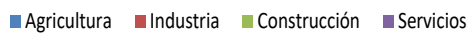

Fuente: elaboración propia.

\section{Gráfico 2: Distribución de las empresas de la muestra según} tamaño.

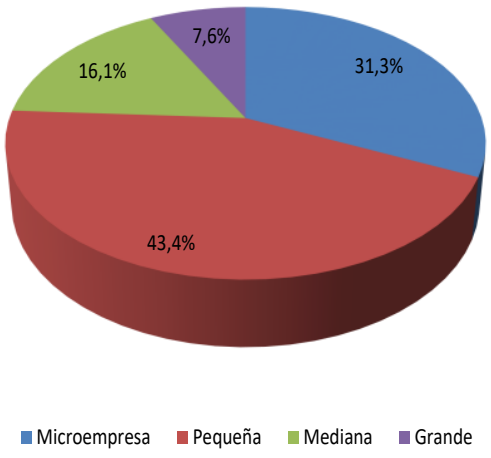

Nota: Microempresa (menos de 10 trabajadores); Pequeña (10-49); Mediana (50-199); Grande (200 o más)

Fuente: elaboración propia. 
Territorialmente, el ámbito de inaplicación fundamental fue la provincia ( $86 \%$ de los casos), seguido a gran distancia por la comunidad autónoma (11\%), adquiriendo el ámbito nacional una importancia residual (3\%). En el gráfico 3 se recoge la información relativa al contenido de las inaplicaciones, pudiéndose observar que la cuantía de los salarios a cobrar por los trabajadores fue la cláusula de inaplicación más frecuente. Prácticamente todas las empresas que inaplicaron (el $94,2 \%$ ) lo hicieron sobre esta cláusula. Adicionalmente, un $20 \%$ de las empresas inaplicaron contenidos relacionados con el sistema de remuneración (téngase en cuenta que las empresas pueden aplicar simultáneamente varias de las condiciones pactadas en convenio). En un orden de importancia ya mucho menor se encuentran aspectos relacionados con la jornada de trabajo (número de horas y su distribución), la organización del trabajo en la empresa, y aspectos relacionados con la Seguridad Social.

\section{Gráfico 3: Condiciones de trabajo que se inaplican.}

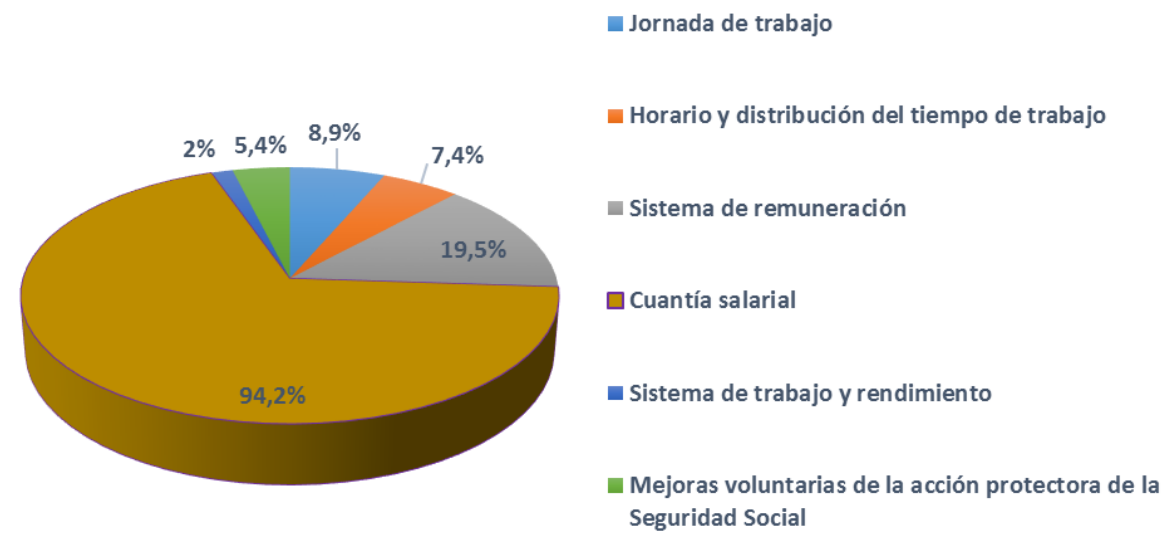

Fuente: elaboración propia.

En cuanto a la duración de las inaplicaciones, el 28\% de las empresas acordaron inaplicar estas cláusulas durante un año o menos, el 30\% entre uno y dos años, y el 16\% durante más de dos años. También hay que destacar que en un porcentaje elevado de los casos (26\%) la fuente estadística (Registro de Convenios) no indica el periodo de inaplicación.

Por último, analizando la evolución en el periodo 2007-2014 de varios indicadores económicos de las empresas de la muestra, podría 
parecer que el hecho de descolgarse del convenio en 2012 o en 2013 ha ayudado en muchos casos a la recuperación de la actividad, del empleo y de la rentabilidad económica de las mismas. Así en el gráfico 4 se recoge la evolución de los ingresos de explotación, los resultados después de impuestos, y la rentabilidad económica. En general, después de unos primeros años de crisis en los que tanto los ingresos de explotación, como los resultados y la rentabilidad económica se habían derrumbado, se observa una recuperación de los tres indicadores a partir del año 2012, primero en el que las empresas pueden acudir legalmente al recurso de la inaplicación. Sin embargo, esta tendencia no dista mucho de la observada para el conjunto de empresas de la economía (las que inaplican y las que no) a partir de 2012. En concreto, en el gráfico 5 se muestra la evolución del índice general de evolución de la cifra de negocios empresarial de la economía española en el periodo 2007-2014, proporcionada por el INE. En él se observa que la caída de la cifra de negocios es continuada hasta 2012 (a diferencia de lo que se observa en el gráfico 4, en el que se detecta un primer intento de recuperación en 2010 y 2011), pero a partir de 2012 se produce una elevación de la cifra de negocio para el conjunto de empresas de la economía, muy similar al aumento de los ingresos de explotación de las empresas que inaplican el convenio.

Por su parte, el gráfico 6 muestra la evolución del nivel de empleo y del coste laboral medio anual para las empresas de la muestra durante el mismo periodo (2007-2014). ${ }^{9}$ En este caso, las dinámicas no son totalmente similares. Si bien parece que las inaplicaciones han permitido a las empresas elevar sus niveles de empleo desde el año 2013 y especialmente en 2014, la evolución del coste laboral medio ha sido peor, recuperándose sólo en 2014. Este resultado es totalmente lógico, pues cómo ya se ha visto, el $94 \%$ de las empresas inaplican la cuantía salarial (esto es, reducen el salario medio), y es precisamente este abaratamiento de costes laborales lo que ha podido tener una incidencia positiva sobre el empleo. Si comparamos esta evolución con la correspondiente al conjunto de la economía española (gráfico 7), se observan algunas diferencias. En concreto, para el conjunto de la economía el número de ocupados comienza a recuperarse con un año de retraso (en 2014), y el coste laboral medio sigue cayendo en ese año. Parece que, frente a la dinámica general, las empresas que inaplican el convenio colectivo aceleran un año la recuperación.

\footnotetext{
${ }^{9}$ Hemos de señalar que la base de datos SABI no distingue entre empleados indefinidos o temporales, por lo que no se puede desglosar la evolución del número de empleos en estas dos categorías.
} 
Gráfico 4: Evolución de cifras de negocio para las empresas que inaplican (2007-2014).

\section{Ingresos de explotación (millones de euros)}

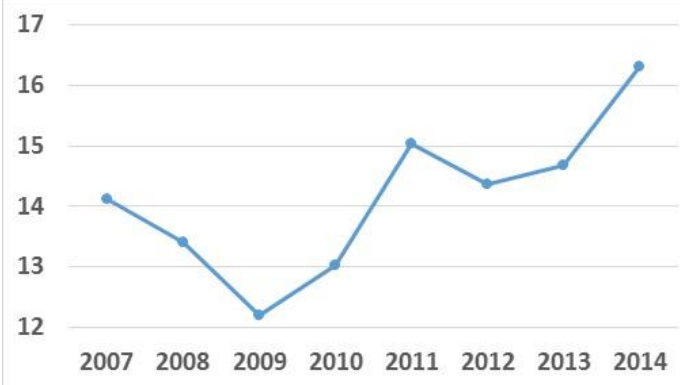

Resultados después de impuestos (miles de euros)

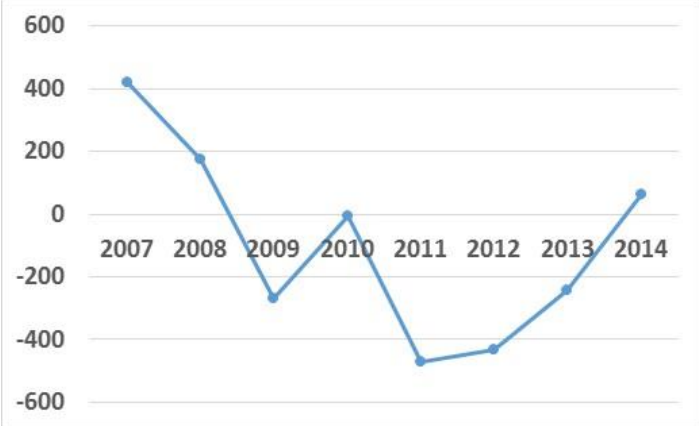

\section{Rentabilidad económica (\%)}

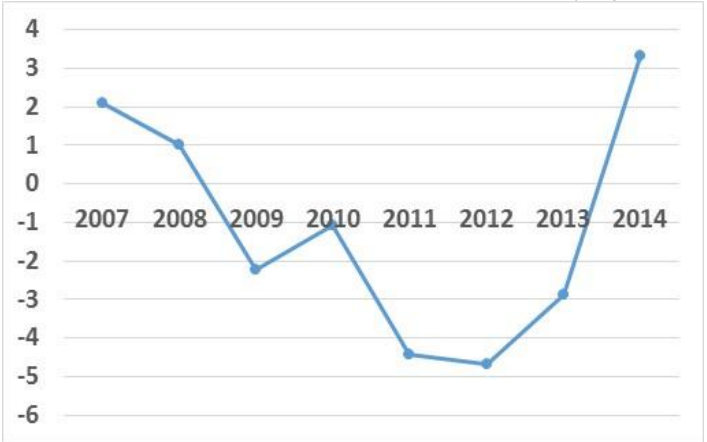

Fuente: elaboración propia. 
Gráfico 5: Evolución del índice general de cifra de negocios empresarial de la economía española, corregido de efectos estacionales y de calendario (2007-2014).

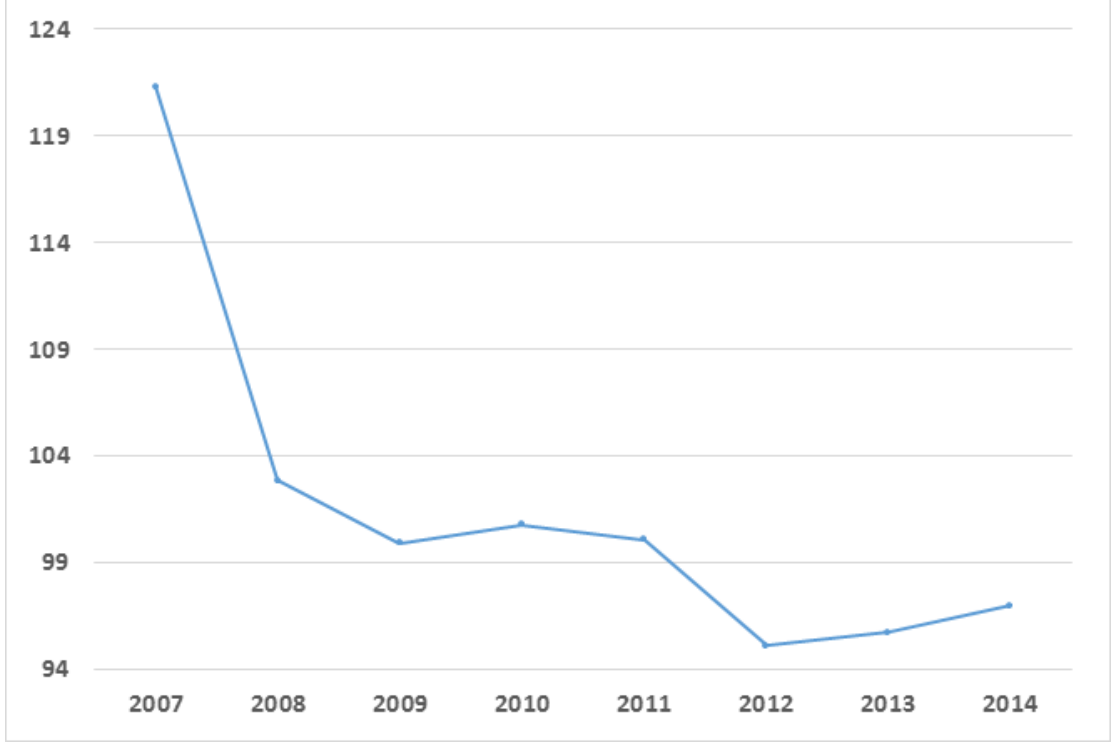

Fuente: INE. 
Gráfico 6: Evolución del volumen de empleo y del salario medio anual (2007-2014).
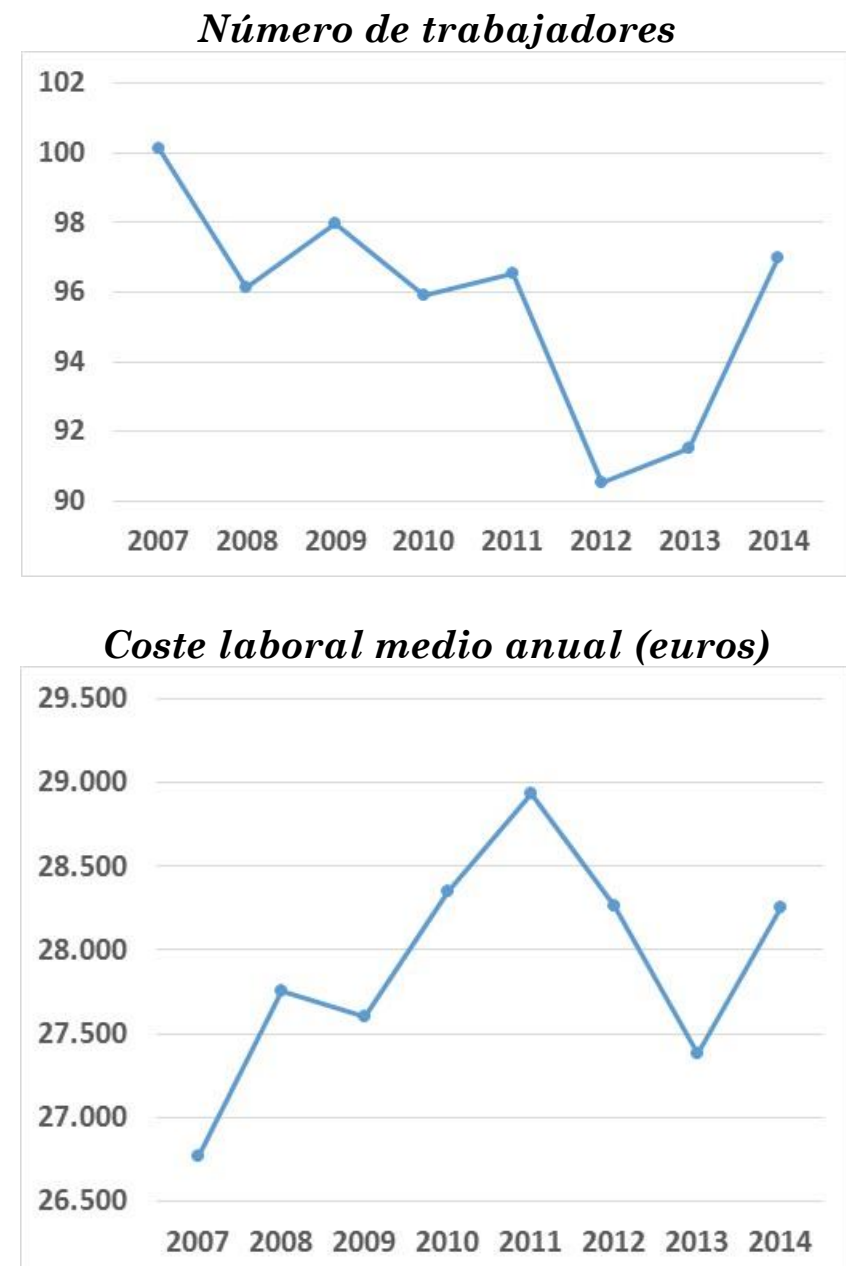

Fuente: elaboración propia. 
Gráfico 7: Evolución del volumen de empleo (2007-2014) y del salario medio anual (2008-2014) para el conjunto de la economía española.

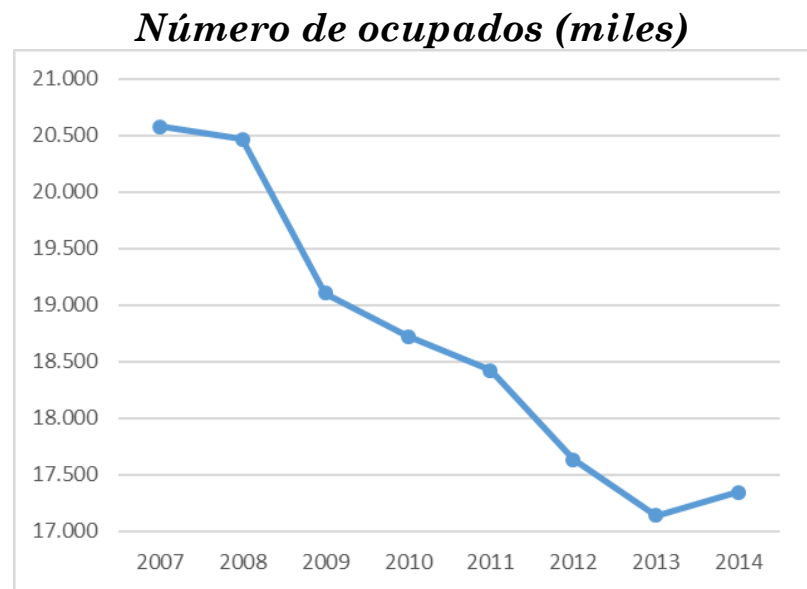

Coste laboral medio anual bruto (euros)

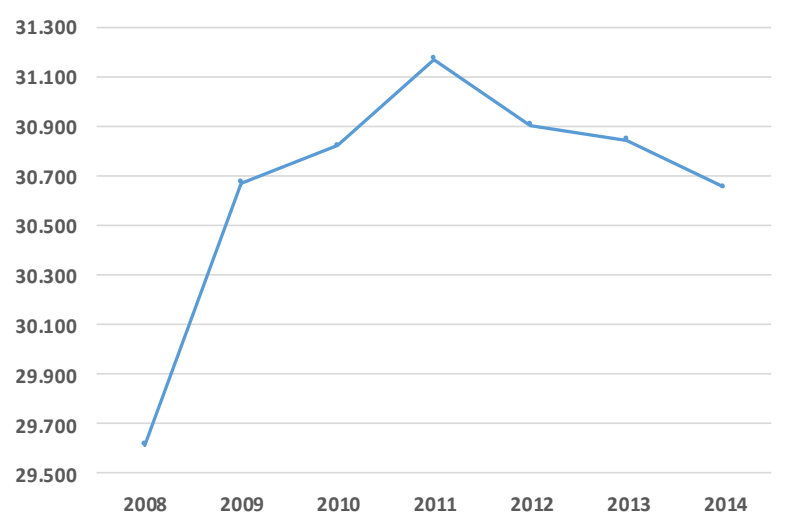

Fuente: EPA-INE y Encuesta Anual de Coste Laboral-INE. 


\subsection{Determinantes de la evolución del empleo de las empresas que inaplican}

Como ya se ha señalado, la inmensa mayoría de las inaplicaciones se centran en las cláusulas salariales de los convenios. Inaplicar implica, pues, reducir costes laborales con mayor o menor intensidad. Por este motivo, tiene interés incidir aún más en la cuestión de si esta reducción de costes laborales ha podido tener un impacto positivo sobre el empleo de las empresas que inaplican, al margen del efecto de otras variables como puede ser la evolución del ciclo económico con posterioridad a 2012. En otras palabras, se trataría de averiguar si la flexibilidad de costes aportada por el nuevo marco legal de las inaplicaciones ha favorecido la recuperación del empleo para las empresas en crisis. Para ello, utilizando la muestra de empresas que inaplican el convenio, se procede a estimar el efecto sobre la tasa de variación del empleo entre 2013 y $2014, \vec{L}$, de un conjunto de variables explicativas de la evolución de dicha tasa. Por un lado, dado que la demanda de trabajo es una demanda derivada de la producción o ventas de la empresa, entre las variables explicativas se incluye la tasa de crecimiento de los ingresos de explotación, tanto la correspondiente al periodo $2013-2014, \dot{Y}$, como la correspondiente al periodo anterior, 2012-2013, $\dot{Y}_{-1}$. Esta última variable se incluye con el objetivo de comprobar si el empleo de las empresas pueda verse afectado también por la evolución de los ingresos en el pasado inmediato, y no sólo en el periodo corriente. Se espera que el efecto de estas variables sobre el empleo sea positivo y significativo. Por otra parte, la evolución de la cantidad demandada de trabajo de una empresa está influida negativamente por la evolución del precio del trabajo, por lo que se ha de incluir entre las variables explicativas la tasa de variación del coste laboral medio, $W$. En tercer lugar, además de estos factores internos a la empresa, hay elementos externos que tienen que ver con la dinámica del ciclo económico y que pueden condicionar la evolución del empleo. En nuestro caso, hemos elegido la tasa de variación del PIB regional como proxy de la influencia del ciclo económico en la marcha de la empresa (tanto la correspondiente al periodo 2013-2014, PIBR, como la correspondiente al periodo anterior, 2012-2013, $P I B R_{-1}$ ). Adicionalmente, como elementos de control se han introducido las siguientes variables, cuya información se ha obtenido para cada empresa del Registro de Convenios Colectivos del Ministerio de Empleo y Seguridad Social: 
a) Sector al que pertenece la empresa: agricultura, construcción y servicios (siendo la industria la categoría de referencia).

b) Año de registro de la inaplicación (la variable dicotómica inaplica2013 toma valor 1 cuando la empresa inaplica el convenio en 2013 y 0 si lo hace en 2012).

c) Duración de la inaplicación. Se distinguen los siguientes tramos a través de un conjunto de variables dicotómicas: de 0 a 6 meses (inaplica0a6), de 6 a 12 (inaplica6a12), de 12 a 18 (inaplica12a18), de 18 a 24 (inaplica18a24), más de 24 meses o no definida (inaplica>24), siendo esta última la categoría de referencia.

d) Tipo de inaplicación. Las empresas pueden inaplicar varias cláusulas de un convenio colectivo de manera simultánea. El Registro de Convenios Colectivos indica, para cada empresa seleccionada, si se han producido las siguientes inaplicaciones, a partir de las cuales se construyen distintas variables dicotómicas: inaplicación de jornada (inapjornada); de horario (inaphorario); de sistema de remuneración (inapsisrem); de cuantía salarial (inapsalario); de sistema de trabajo (inapsistrab); y de mejoras en la Seguridad Social (inapmejorass). Cabe recordar de nuevo que el 94\% de las empresas de la muestra inaplican la cuantía salarial. Claramente, el objetivo central de las inaplicaciones consiste en reducir el coste laboral de las empresas.

e) Ámbito del descuelgue. Se distinguen distintos ámbitos de descuelgue a través de un conjunto de variables ficticias: ámbitofranja, si el descuelgue se produce sólo para un segmento de los empleados de la empresa; ámbitocentro, si se aplica sólo a un centro de trabajo de la empresa; y ámbitoempresa, si la inaplicación se produce para el conjunto de la empresa (opción mayoritaria que tiene lugar en el $86 \%$ de los casos y que se toma como categoría de referencia).

En definitiva, el modelo a estimar es el siguiente:

$\dot{L}=\mathrm{f}\left(\dot{Y}, \dot{Y}_{-1}, \dot{W}, P I B R, P I B R_{-1}\right.$, Sector, Año de registro de la inaplicación, Duración de la inaplicación, Tipo de inaplicación, Ámbito del descuelgue) 
El modelo se ha estimado por Mínimos Cuadrados Ordinarios, y los resultados de la estimación aparecen en el cuadro $5 .{ }^{10} \mathrm{Al}$ estar definido el modelo en tasas de crecimiento para las variables empleo, ingresos de explotación, PIB regional y coste laboral medio, los coeficientes estimados pueden interpretarse como elasticidades. Por ejemplo, la elasticidad del empleo ante cambios en el coste laboral medio es -0,37. Por tanto, una reducción del $1 \%$ en el coste laboral medio de las empresas se traduce en un aumento del $0,37 \%$ en el empleo. La variación en el empleo también se muestra sensible a la tasa de crecimiento de los ingresos de explotación de la empresa en el periodo corriente y en el anterior (con unas elasticidades de 0,53 y 0,010 respectivamente). En cuanto al efecto del ciclo económico regional, se observa que el empleo responde más a la tasa de variación del PIB regional del periodo anterior que a la del actual. Esto es, mientras que los cambios que se producen en los factores internos a la empresa, como los ingresos y los salarios, se manifiestan de forma inmediata en el empleo, los efectos del ciclo económico general se manifiestan sobre el empleo con un periodo de retraso. Por lo demás, el resto de variables de control incluidas en la estimación no resultan significativas, con la excepción de la variable inaplica0a6. Es decir, frente a la categoría de referencia, que es inaplicar el convenio para más de 24 meses o por un tiempo que no se define en la estadística, el hecho de inaplicar por un periodo de seis meses o menos reduce la tasa de variación del empleo. En otras palabras, las inaplicaciones de menor duración son las que tienen menos éxito de cara a recuperar el empleo de las empresas afectadas por la crisis que han decidido inaplicar. Obsérvese que los tipos de cláusulas que se inaplican no introducen diferencias en la tasa de variación del empleo de las empresas, quizá porque la inmensa mayoría de las mismas, como ya se ha indicado, inaplican la cuantía salarial. Adicionalmente pueden inaplicar cláusulas de jornada, horario, etc., pero en todo caso, el elemento clave es que el $94 \%$ de las empresas dejan de pagar los salarios de convenio, consiguiendo reducciones efectivas de sus costes laborales.

\footnotetext{
10 Obsérvese que el número final de empresas consideradas en la estimación es de 382. La razón de la diferencia entre el tamaño de la muestra inicial (447 empresas) y esta cifra, es que no se dispone del dato de ingresos correspondiente a 2014 para todas las empresas, debido al retraso con que SABI ofrece sus cifras en algunos casos.
} 
Cuadro 5: Estimación de los determinantes de la tasa de variación del empleo. Mínimos Cuadrados Ordinarios.

\begin{tabular}{|c|c|c|}
\hline Variables & Coeficiente & t-Robusto \\
\hline Constante & 10,141 & 0,98 \\
\hline$\dot{Y}$ & 0,533 & $4,45^{* *}$ \\
\hline$\dot{Y}_{-1}$ & 0,010 & $2,50 * *$ \\
\hline$\dot{W}^{+}$ & -0.374 & $-3,48^{* *}$ \\
\hline$P I \dot{B} R$ & 0.490 & 0,13 \\
\hline$P I B R_{-1}$ & 6,192 & $3,03^{* *}$ \\
\hline Agricultura & 3,761 & 0,61 \\
\hline Construcción & 10,694 & 1,20 \\
\hline Servicios & 2,043 & 0,55 \\
\hline Inaplica2013 & $-2,499$ & $-0,92$ \\
\hline Inaplica0a6 & $-7,622$ & $-1,66^{*}$ \\
\hline Inaplica6a12 & 1,989 & 0,60 \\
\hline Inaplica $12 \mathrm{a} 18$ & $-0,920$ & $-0,22$ \\
\hline Inaplica18a24 & 2,965 & 0,62 \\
\hline Inapjornada & -0.829 & $-1,33$ \\
\hline Inaphorario & 0,601 & 0,16 \\
\hline Inapsisrem & $-0,369$ & $-0,12$ \\
\hline Inapsalario & 0,538 & 0,18 \\
\hline Inapsistrab & $-6,787$ & $-1,44$ \\
\hline Inapmejorass & 2,129 & 0,28 \\
\hline Ámbitofranja & $-6,154$ & $-1,04$ \\
\hline Ámbitocentro & $-3,623$ & $-1,06$ \\
\hline $\mathrm{F}(21,360)$ & 5,47 & \\
\hline $\mathrm{R}^{2}$ & 0,415 & \\
\hline Número de observaciones & 382 & \\
\hline
\end{tabular}

$(* *)$ Significativo al 5\%; $\left(^{*}\right)$ Significativo al $10 \%$

\section{Conclusiones}

La reforma laboral de 2012 incorporó por primera vez mecanismos sencillos de inaplicación de un convenio colectivo. Basta con acreditar una disminución persistente del nivel de ingresos o ventas de una empresa, para que ésta pueda descolgarse de las tablas salariales y de otros aspectos relevantes del convenio, como la jornada de trabajo y el horario. Con ello se pretende, básicamente, que las empresas en crisis puedan ajustar los costes salariales para evitar que los recortes del empleo sean más profundos. A pesar de las facilidades 
que confiere la legislación desde el año 2012, las inaplicaciones no han sido muy frecuentes en la práctica. En general, el porcentaje de trabajadores afectados por las mismas se ha situado por debajo del 1\% del total de trabajadores sujetos a convenio, con la única excepción del año 2013, en el que esta cifra se elevó al 1,5\%. La mejora económica observada desde el año 2014 ha hecho que la cifra de ventas de las empresas tienda a recuperarse, y que, por consiguiente, sean cada vez menos las empresas que pueden hacer un uso legal de las inaplicaciones.

Dado que la finalidad de las inaplicaciones es, fundamentalmente, reducir costes salariales, no es extraño observar que más del $90 \%$ de las mismas se centran en aspectos que tienen que ver con los salarios y con el sistema de remuneración. La cuestión clave que tratamos de analizar en este estudio es saber si las empresas que han inaplicado han conseguido realmente mejorar su situación económica tras llevar a cabo esta medida. Para ello, utilizando la información procedente del Registro de Convenios Colectivos del Ministerio de Empleo y Seguridad Social y de la base de datos SABI (Sistema de Análisis de Balances Ibéricos), se ha seleccionado una muestra de 447 empresas que han inaplicado un convenio en el periodo 2012-2013, observando para todas ellas la evolución entre 2007 y 2014 de los ingresos de explotación, los resultados después de impuestos, los gastos de personal, el número de empleos, el coste laboral medio, y el índice de rentabilidad económica. Se ha podido comprobar que tras el profundo deterioro de los ingresos de explotación, los resultados y la rentabilidad económica que tuvo lugar para estas empresas a comienzos de la crisis, los tres indicadores mencionados se ha ido recuperando ligeramente a partir de 2012, primer año en que las inaplicaciones fueron posibles. Lo mismo ha sucedido, con un año de retraso, con el nivel de empleo, aunque no con el coste laboral medio, que sólo ha comenzado a recuperarse a partir de 2014. Comparando la evolución de estos indicadores con la correspondiente al conjunto de empresas de la economía, parece que si bien la evolución de los ingresos de explotación a partir de 2012 no dista mucho de lo observado en el ámbito general, la recuperación del empleo y del coste laboral parece haberse iniciado con un año de antelación en el caso de las empresas que inaplicaron el convenio. También se ha podido constatar que las empresas que inaplicaron presentan una elasticidad del empleo ante cambios en los salarios de -0,37, por lo que una reducción en el coste laboral medio del 1\% para estas empresas conduce, en promedio, a un aumento del 0,37\% en su nivel de empleo. Sería interesante conocer el valor de esta elasticidad para el mismo conjunto de empresas en un 
periodo anterior, para ver en qué medida se ha modificado dicho valor como consecuencia del cambio legislativo; pero no se dispone de esa información, por lo que este último resultado debe interpretarse con la debida cautela.

\section{Bibliografía}

Bentolila, S. y Jimeno, J. F. (2002). La reforma de la negociación colectiva en España. Documento de Trabajo 2002-03. FEDEA.

Del Rey Guanter, S. (2014). Estructura de la negociación colectiva y prioridad del nivel de empresa tras la reforma laboral de 2012. Revista del Ministerio de Empleo y Seguridad Social. N ${ }^{\circ}$ Extraordinario-La Reforma Laboral de 2012 y su desarrollo: 201-213.

Pérez Infante, I. (2015). Las reformas de la negociación colectiva y la devaluación salarial. (Mimeo).

Segura, J. (2001). La reforma del mercado de trabajo español: Un panorama. Revista de Economía Aplicada, IX (25): 157-190.

Thibault Aranda, J. (2014). La inaplicación del convenio colectivo después de la reforma de 2012. Revista del Ministerio de Empleo y Seguridad Social. N ${ }^{\mathrm{o}}$ Extraordinario-La Reforma Laboral de 2012 y su desarrollo: 215-231. 